\title{
Analyzing the social networks of high- and low-performing students in online discussion forums
}

\begin{abstract}
An ego network is an individual's social network relationships with core members. In this study, the ego network parameters in online discussion spaces of high- and low-performing students were compared. The extent to which students' ego networks changed over the course were also analyzed. Participation in 7 weeks of online discussions was analyzed for 12 highperforming students and 9 low-performing students. Results suggested that ego networks' compositions of high- and low-performing students were significantly different. Particularly, high-performing students had denser ego networks and tended to exhibit a higher level of centralities than low-performing students. Results of network visualization indicated that high-performing students increased and kept their networks stable over the course in comparison to low-performing students, who had fragmented networks. Several networks' change mechanisms for high- and low-performing groups are also identified and discussed.
\end{abstract}

Keyword: Social networks; High- and low-performing; Students; Online discussion forums 